\title{
How to Apply and Implement BIM Technology and Knowledge in Engineering Projects
}

\author{
Fengyi Ding \\ ${ }^{1}$ An De college, Xi' an University of architecture and technology, Xi'an, Shanxi, 710311, China.
}

\begin{abstract}
In the current construction projects, in view of the influence of construction site and external factors, problems such as confusion of technology, poor information and inappropriate communication appeared. These problems directly hindered the construction projects. However, BIM's appearance has greatly improved part of the perplexities. BIM is a new tool and methods in construction business, also civil engineering. Using of BIM technology in construction projects might promote the development of real estate industry.
\end{abstract}

\section{Introduction}

Since problems mentioned in the abstract, the paper mainly on elaborates how to apply and implement BIM technology and knowledge in engineering projects from different angles. In addition, the application of BIM technology in Water Cube is illustrated. More than this, some difficulties, problems and solutions that might be encountered are also mentioned.

\section{BIM has some main features that can not be replaced by traditional drawing methods}

In recent years, due to the influence of national policies, the development of China's construction industry has slowed down, but the output value of the construction industry still accounts for a large proportion of our national economy. Taking 2018 as an example, China's GDP in 2018 was 90,030.95 billion yuan. Among them, the total output value of the construction industry is 23.50855 billion yuan, and the total output value of the construction industry accounts for $26 \%$ of GDP. Since the large demand in the construction industry, improving efficiency has become the core of construction engineering. The application of BIM makes this problem well solved.

Table1. The construction industry output value proportion of GDP

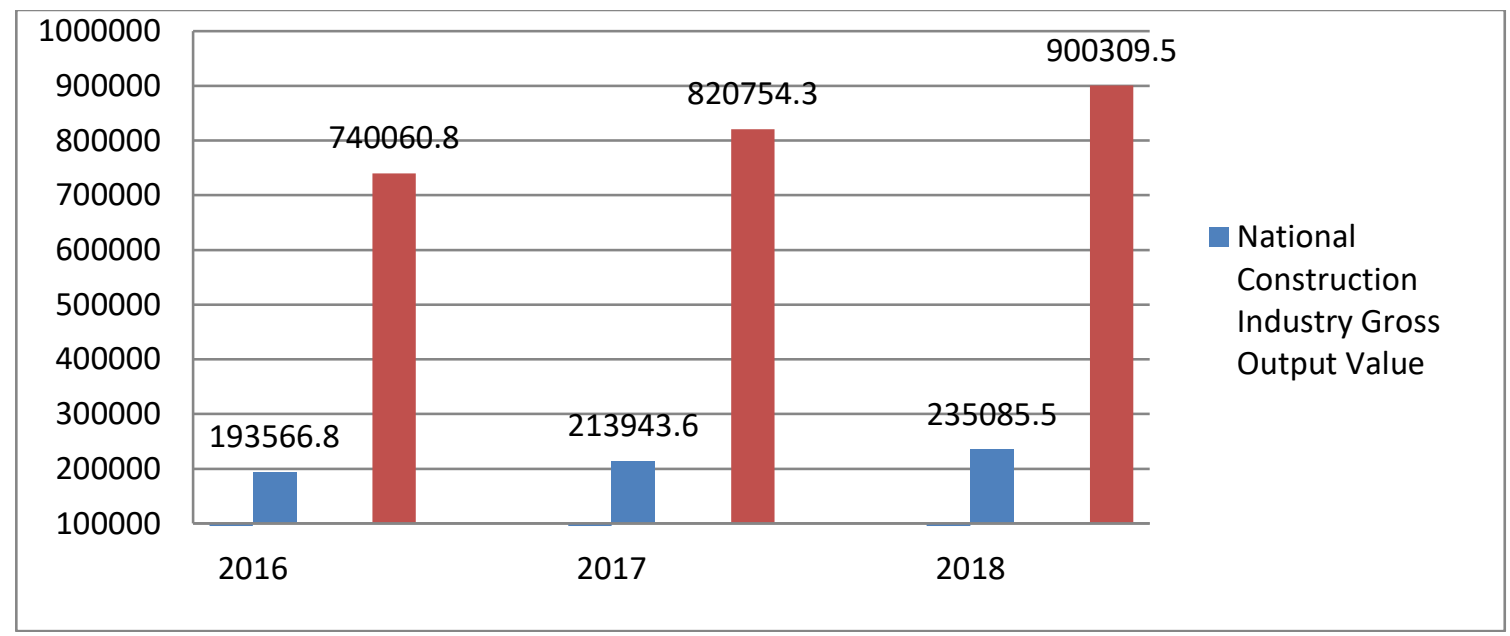

Resource: China Statistical Yearbook 


\subsection{The concept of BIM}

BIM technology is a multi-dimensional (three-dimensional space, four-dimensional time, five-dimensional cost, $\mathrm{N}$-dimensional more applications) model information integration technology, it improve work efficiency, save resources, reduce costs and achieve sustainable development. More than this, it is able to make all elements in construction projects such as government authorities, owners, design, construction, supervision, cost, operation management, project users, etc. from concept generation to completion in the project. What's more, risk objectives is fairly helpful for building information. From design to construction and operation of buildings to the end of the whole life cycle of buildings, integration is realized. All kinds of information are always integrated into 3D model information database. That is to say, it improve efficiency of your work and decrease errors in construction projects. People can work together based on BIM. In addition, you can operate the information and the model by the model. Therefore, fundamentally adjust the method employees rely on symbolic and written drawings for project construction and operation management during the whole life cycle of demolition. BIM makes the whole design process intuitive and clear.

\subsection{Special features can not be replaced by traditional drawing methods}

\subsection{1.visualization}

The application of visualization is fairly significant in construction industry. Here is an example, construction drawings are often obtained, but each element's information is expressed by line drawing. Also, visual methods with thinking is known in BIM, which means designers are capable of having a three-dimensional physical graphics of the components.

\subsubsection{Simulation}

BIM simulate the stuff which need to be simulated in emergency evacuation simulation, design and etc, heat conduction simulation are included. Then it carry out 4D simulation in the bidding and construction stage. In other words, according to construction's organization, actual construction is simulated for a reasonable construction plan determining to guide the construction.

\section{Application \& Cases Analyze of BIM}

\subsection{Application of BIM in Construction}

According to Stanford University Integrated Facility Engineering Center conducted a survey on BIM applications in 2007. Here are results, it is obvious that the advantages of BIM are gradually recognized.
Table 2 BIM in construction engineering application value

\begin{tabular}{|cc|}
\hline Benefits of using BIM & Results \\
\hline Save investment estimation time & $80 \%$ \\
\hline Save off-budget changes & $40 \%$ \\
\hline Save total project investment & $10 \%$ \\
\hline Save project time & $7 \%$ \\
\hline Controlling Investment Estimation Errors & $3 \%$ \\
\hline
\end{tabular}

\subsubsection{Building component modeling}

To begin with, the whole construction project is decomposed into different components because of the building drawings, and their dimensions, the three-dimensional component model measure through volumes and weights as well as the type and type of materials used. Furthermore, in the light of the primary components, facilities of construction and tools are selected to determine the construction method. Through building component modeling, it is useful for builders to learn how to construct on site in advance.

\subsubsection{Multi disciplinary coordination}

The organization and cooperation between professional subcontractors is an important factor to improve the construction progress, and also the key to the smooth implementation of the project. At present, due to the lack of coordination among HVAC, fire protection, strong and weak electricity and other specialties because the affect on technical differences, construction site, professional coordination and other factors, there are inevitably many local, hidden and unpredictable problems, which easily lead to the intersection of various specialties in some building planes and elevation positions. Fork, overlap, unable to work according to the construction drawings. Through the visualization, parameterization and intellectualization of BIM technology, multi-specialty collision inspection, net height control inspection and precise pre-embedding, or using 4D construction management based on BIM technology to pre-simulate construction process and coordinate the various specialties according to the difficulties, measures such as technical errors and ditches can be reduced. Coordination problems brought about by mistakes decrease rework and save costs.

\subsubsection{Site layout optimization}

The appearance of BIM technology gives a good method for layout work. In each stage, site layout should be flexibly carried out to achieve reasonable and efficient site. By applying the equipment and facilities family resources of the project site, after creating the project site model and building model, the actual environment around the project and the site is linked to the model in the form of data information, and the three-dimensional field ground layout is set up. 


\subsubsection{Cost accounting}

More than this, some tough problems which can not be deal with, BIM provides a good opportunity, such as cost accounting. Here are some ways of BIM to make cost accounting easier and efficient.

(1)Create a BIM based actual cost database

Establishing the 5D relational database of cost, let the actual cost data enter the 5D relational database in time, and the cost summary, statistics and split can be obtained immediately. The actual cost BIM with the unit price of labor, material and machine of each WBS unit quantity as the main data. For items without unit price determined in the contract, enter first according to budget price. When there is actual cost data, replace it according to the actual data in time.

\section{(2)Real cost data enters the database in time}

To start with, the cost data in the actual cost BIM is based on the contract price and the enterprise quota consumption. With the progress of the schedule, there will be differences between the actual consumption and the quota consumption, which are supposed to be adjusted in time. Check the actual consumption every month and adjust the actual cost data. Break it up into parts, dynamically maintain the actual cost BIM, greatly reduce the one-time workload, and help to ensure the accuracy of data.

\subsection{Application of Water Cube}

Due to primary features of BIM, it can be used in these aspects in the process of construction engineering, building component modeling and multi disciplinary coordination. Then I will use the case study of BIM Application in Water Cube of National Swimming Center to illustrate them.

\subsection{1.structural design}

The structure depends on the effective sub-division of three dimensional space fundamental arrangement of cells and the soap bubbles. Therefore, Nature beats technology since it is extremely repetitive and highly buildable-removing the usual architectural inspiration in building design. Arup put forward by Professors Weaire and Phelan in Trinity College Physics to deal with Lord Kelvin's conundrum.

\subsubsection{The geometric shape}

The structure of the building is based on a repeating unit consisting of two 12 -sided polyhedrons and six 14-sided polyhedrons. This repeat as a unit in 3D space. The whole building cover with ETFE foil cushions, that is to say it It has prefect insulation, not only this, greenhouse effect is caused. In addition, $20 \%$ of the solar energy is trapped in it, used to heat the interior area.

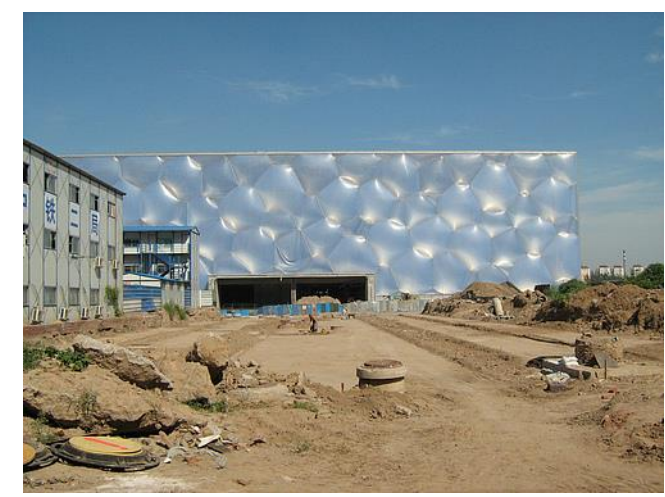

Resource: www.mipang.com

Fig 1 ETFE foil cushions

\subsubsection{The model}

Exporting the Micro Station 3D model to an STL file to create a rapid prototype model might meet the needs of modern Sports venues. A process called SLA may set up the model. Therefore, during the process, liquid epoxy resin is solidified by a laser following the STL file information to get a plastic model.

\section{Some problems that need improvement}

To begin with, the weakness and promotion hindrance lies in the efficiency and performance of hardware and software itself. Autodesk focuses on the interoperability of various three-dimensional software formats, and proposes ask universal format to make the interaction compatibility between software models more convenient, and even some of them can be interoperable in two directions. At this time, a single professional operation fluency can basically meet the use requirements. But with it, the complexity of the project is also increasing. With various but professional applications, the need for collaboration among multiple specialties has been put forward, which has become the main direction of software updating. Later, more complex requirements such as the long-distance real-time interaction between the designer, the owner and the builder were put forward. These aspects of the current speed is not able to achieve. So at any time, there is still a need to improve software performance.

What's more, the second disadvantage is the standardization of software data. Revit is only a basic data set up by BIM in the early stage of design. In the later application, construction and management, a lot of data need to be added. At present, these kinds of software develop data independently and have different structures, which is of course a hindrance to the use of users. But I believe that with the ability of Autodesk, these functions will be integrated in the right time. For example, the energy consumption analysis module has been added in the 2012 edition, dynamo in the 16 edition and form it in the 17 edition. It is believed that there is no difficulty in adding such functions as statistical engineering quantity and cost. Autodesk has the ability to connect the whole 
design chain data. More than this, it also have some problems need to be solved such as insufficient BIM manpower requirements, insufficient motivation and pressure for designers to change. All of the above factors will affect the development of BIM in China, but according to the experience of foreign examples, it can be found that the implementation of the BIM mode does have a clear improvement in the efficiency of construction and mechanical and electrical engineering. Down to the construction factory can jointly make the BIM model work together. When the integrity and correctness of the drawing elements can be greatly improved, the model accuracy rate is naturally closer to the real. The control of materials and the integration of pipelines will also be clearer, which will make the mastering of work schedules more accurate.

\section{Conclusion}

Generally speaking, the application of BIM is Building component modeling, multi disciplinary coordination, and site layout optimization. BIM is pretty useful and perhaps to instead of the traditional methods someday. Therefore, there are more and more buildings which designed by BIM, and fixed by BIM. Just as Water Cube of National Swimming Center, which was mentioned in this paper. In the past, because of popularization of CAD technology, architects can free their hands. This is the first revolution in engineering and design. At present, the emergence of BIM promotes the second revolution in the entire construction field. BIM brings the progress and renewal of existing technology. More than this, it influent ways of people to think about in the future. In summary, I hope China government can request to use BIM widely as soon as possible.

\section{Acknowledgments}

This thesis would not have been possible without the support of my lecturer, professor Gao, and also my parents, they give me support when I got into troubles. I would like to take this chance to extend my sincere gratitude to these persons who have helped me during the thesis-writing.

\section{References}

1. HongLiang, Y. YiHua,M. (2014) Project management. Zhejiang University Press, Hangzhou.

2. MinXin, H. HuaiJian, L. (2015) Civil Engineering Drafting 4th Edition. Wuhan University of Technology Press, WuHan.

3. Da Li, 2018, Typical applications of BIM in China's construction engineering industry. https://wenku.baidu.com/view/039cd932842458fb77 0bf78a6529647d27283495.html

4. LiuChang. (2014) Research on cost management in the whole process of construction project based on BIM. Chongqing University, Chongqing.
5. Congpeijing. (2006) project management. China Construction Industry Press, Beijing. 